\title{
Neural network multispectral satellite images classification of volcanic ash plumes in a cloudy scenario
}

\author{
Matteo Picchiani ${ }^{1 *}$, Marco Chini ${ }^{2}$, Stefano Corradini ${ }^{3}$, \\ Luca Merucci ${ }^{3}$, Alessandro Piscini ${ }^{3}$, Fabio Del Frate ${ }^{1}$ \\ 1 Tor Vergata University, Dipartimento di Ingegneria Civile \\ e Ingegneria Informatica, Rome, Italy, 2 Luxembourg Institu- \\ te of Science and Technology, Environmental Research and \\ Innovation Department, Belvaux, Luxembourg, 3 Istituto Na- \\ zionale di Geofisica e Vulcanologia, Roma, Italy \\ *picchian@disp.uniroma2.it
}

\begin{abstract}
This work shows the potential use of neural networks in the characterization of eruptive events monitored by satellite, through fast and automatic classification of multispectral images. The algorithm has been developed for the MODIS instrument and can easily be extended to other similar sensors. Six classes have been defined paying particular attention to image regions that represent the different surfaces that could possibly be found under volcanic ash clouds. Complex cloudy scenarios composed by images collected during the Icelandic eruptions of the Eyjafjallajökull (2010) and Grimsvötn (2011) volcanoes have been considered as test cases. A sensitivity analysis on the MODIS TIR and VIS channels has been performed to optimize the algorithm. The neural network has been trained with the first image of the dataset, while the remaining data have been considered as independent validation sets. Finally, the neural network classifier's results have been compared with maps classified with several interactive procedures performed in a consolidated operational framework. This comparison shows that the automatic methodology proposed achieves a very promising performance, showing an overall accuracy greater than $84 \%$, for the Eyjafjallajökull event, and equal to $74 \%$ for the Grimsvötn event.
\end{abstract}

\section{INTRODUCTION}

A fter volcanic activity, ash clouds affect the population and its economy at different levels, and represent a major hazard for air traffic due to the harming effects they have on aircraft engines. Accurate and fast satellite monitoring of the phenomena's evolution is one of the most effective tools when managing the risk mitigation phase. In the late 1980s, Prata demonstrated [1989] how ash particles of effective radius, ranging between about 0.5 to $10 \mu \mathrm{m}$, can be identified by thermal infrared (TIR) radiometers, since at shorter infrared wavelengths ash absorbs more radiation than water droplets or ice, which mainly absorb at longer infrared wavelengths. This behavior implies that the difference between the brightness temperature measured at 11 and $12 \mu \mathrm{m}$ (BTD) will be negative if the Instantaneous Field of View (IFOV) area is contaminated by ash. On the contrary, the BTD will generally be positive for meteoro- 
ANNALS OF GEOPHYSICS, Fast Track 2, 2014

logical clouds. Different works studying the eruption phenomena characterization using different satellite data are based on this assumption [Prata and Kerkmann, 2007; Corradini et al., 2008]. Such studies highlighted the role of remote sensing as a primary source of information when retrieving ash particle properties such as effective radius, optical depth and the total column mass. Moreover, sensitivity analysis highlighted the importance of ancillary information to constrain the output of radiative transfer models and showed that the accuracy of inversion methods is affected by errors even equal to $40 \%$ [Corradini et al., 2008]. The ash retrieval accuracy is also constrained by the emissivity of the surfaces underlying the ash clouds, since ash is semitransparent in the TIR spectral range. Thus, the ash retrieval can generally achieve better results if ash clouds and their underlying surfaces are correctly classified [Corradini et al., 2010]. To identify these classes, a "standard procedure" based on BTD, VIS channels, MODIS Level 1 (L1) Land/Sea mask and MODIS Level 2 (L2) Cloud products is generally applied in a cascade procedure (see Section II). In this paper, we propose a novel classification approach based on Neural Networks (NN) to identify ash clouds and their underlying surfaces, which can recursively be applied in order to quickly obtain automatic and accurate maps. The suitability of the NN approach for the detection of ash cloud in conditions free from meteorological cloud, has already been proved [Picchiani et al., 2011], and the detection was simplified by considering two main classes: "ashy" and "non-ashy" pixels in the MODIS images. Here we propose to classify a more complex scenario, where "ash above sea" and "ash above meteorological clouds" classes are present, both playing an important role in the ash parameters retrieval. The NN algorithm has been tuned on Moderate Resolution Ima- ging Spectroradiometer (MODIS) images collected in overcast conditions during the 2010 Eyjafjallajökull and 2011 Grimsvötn eruptions. This test set represents a challenge in the identification of the ashy pixels. Indeed, the complexity of the images characterized by meteorological formations at different heights and other issues like the presence of glaciers, if not correctly identified, may produce false alarms even with the "standard procedure". Therefore, the NN classifier is also trained to identify the other main surfaces in the image, defining a total of six classes labeled in the following manner: 1-Ash above Sea; 2-Ash above meteorological clouds; 3-Meteorological clouds; 4-Sea surface; 5-Ice surface;. 6-Land.

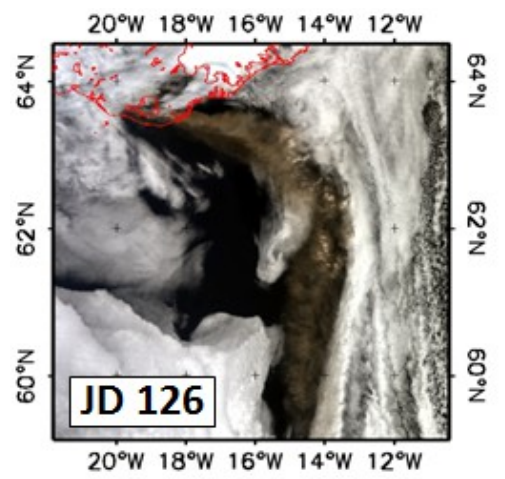

Figure 1: RGB (channels 1-4-3) color composite of MODIS images acquired 6th, May 2010 (JD 126) during the Ejafjallajökull eruption (coastline plotted in red), used to train the NN.

\section{METHODOLOGY}

The most common type of NN, known as feedforward Multilayer Perceptron (MLP) [Bishop, 1995], has been chosen for this study. A MLP is composed of simple non-linear computational units called neurons, sorted in layers and connected by weighted links (synapses). The weights can be considered as parameters of a nonlinear regression computed through a process of error minimization between input in- 
formation and computed outputs, referred to as the training phase. NN classifiers are good candidates for this kind of problem because: 1) non parametric approaches are not limited to prior assumptions on the distribution of input data, thus making the integration of different sources more effective; 2) an output is produced very quickly if the $\mathrm{NN}$ is correctly trained. With regard to the first reason, it is well-known that any classification algorithm, which has to reproduce a physical phenomenon, needs a representative dataset for the training phase and, usually, in real scenarios this requirement partially fails. This is due to outliers or spectrally non-pure pixels. In this respect, the MLP-NNs have already proved to be more robust than parametric classifiers, such as Bayesian ones [Chini et al., 2008]. Regarding the second reason for using NN classifiers, it is already well-known that in an emergency one of the principal requirements is speed, thus this immediate automatic classification of the image could be an effective solution. This approach can be a precious tool and significantly improve the retrieval of ash parameters, especially when the ash cloud moves from one image to the next and the surface under the ash changes (e.g., from sea to land or ice). In this study, the MPL-NN potentials have been exploited to classify three MODIS images collected on $8^{\text {th }}, 12^{\text {nd }}$ and $13^{\text {rd }}$ of May 2010 (JD 128, 132 and 133 respectively) during the Eyjafjallajökull eruption and one collected on $23^{\text {rd }}$ May 2011 (JD 143) during the Grimsvötn eruption. The dataset also includes another image of the Eyjafjallajökull event acquired on $6^{\text {th }}$ May 2010 (JD 126) which is used to train the NN (see Fig. 1). The NN classifier is designed to replicate the same results as those obtained when applying the iterative "standard procedure". The latter is the most suitable approximation to represent the real situation pictured by the sensor.
Therefore, all the MODIS images were first interactively classified, and then the results were used to train and evaluate the NN algorithm. The "standard procedure" uses the BTD approach firstly to identify ash clouds and then to classify them by putting them into "ashy pixels" above sea and "ashy pixels" above meteorological clouds by intersecting the BTD output with both the MODIS L1 Land/Sea Mask and the MODIS L2 Cloud Products. The latter are also used to classify all the other meteorological clouds present in the satellite image. The classes "Land" and "Sea" are obtained by the intersection between the MODIS Land/Sea Mask and the ash and meteorological cloud pixels previously classified. The result of this operation is subtracted from the pixels indicated by the Land/Sea Mask in order to identify the "Land" and "Sea" classes. Finally, the "Ice" surfaces are identified by fixing an appropriate threshold on the MODIS band 7 radiance. As stated before, only one MODIS image has been used here for training, while the others have been used as independent validation sets. This approach was considered in order to assess the generalization capabilities of the NN and the statistical representativeness of the training/test samples. In particular, the validation on the Grimsvötn eruption image represents a very strict test as the data is totally independent from the training set. One of the most critical issues in training complex $\mathrm{NN}$ is the selection of the inputs, since generally fewer inputs ensure better generalization capabilities [Bishop, 1995]. Consequently, a branch and cut approach has been applied in order to select the most significant MODIS channels from the whole set of bands. The selected MODIS channels are: $1 \div 7,20 \div 23,29,31$ and 32. This selection of input wavelengths optimizes also the retrieval approach of ash parameters based on NN [Piscini et al. 2014]. 
ANNALS OF GEOPHYSICS, Fast Track 2, 2014
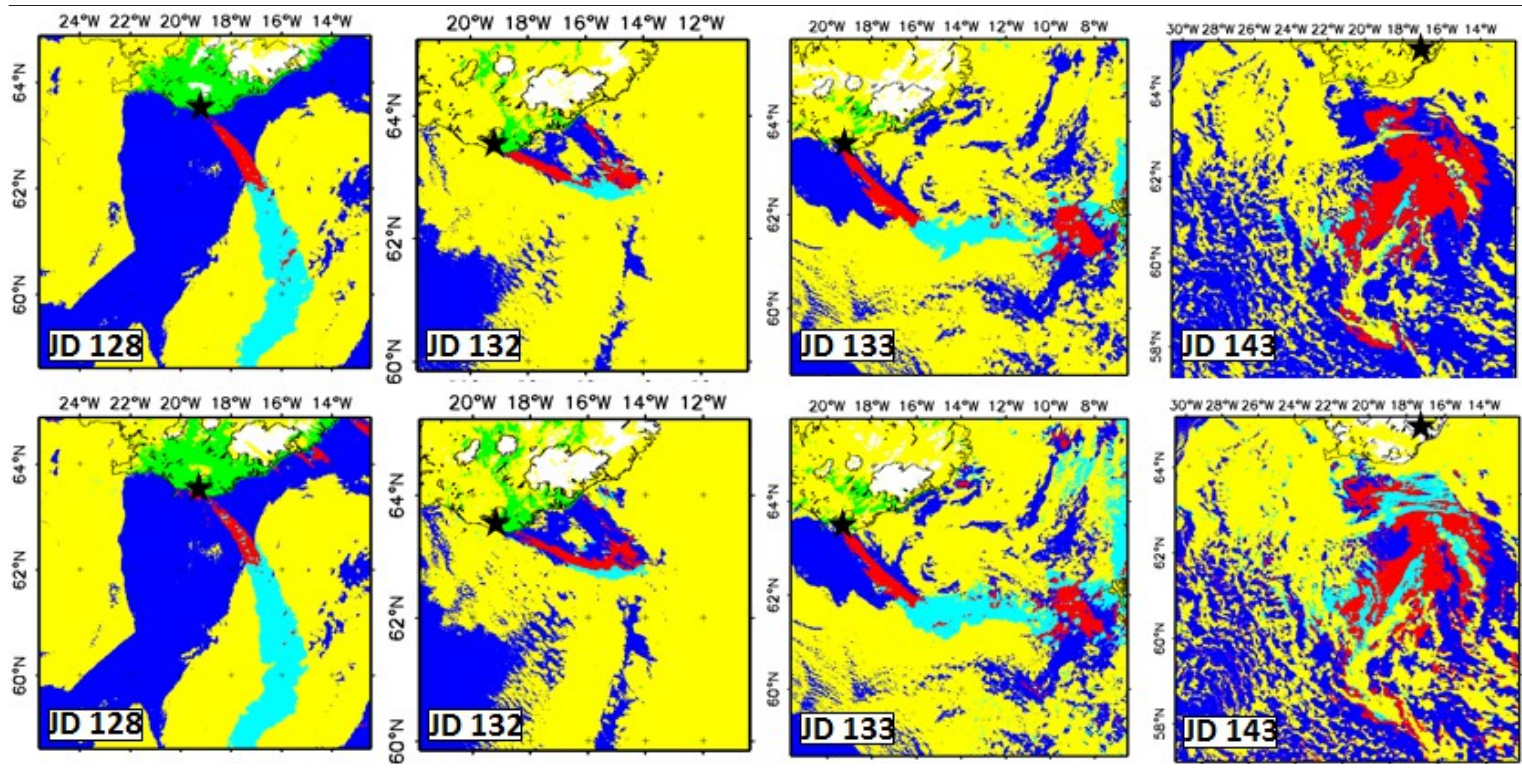

Figure 2: Standard procedure maps (upper panel). NN maps (down panel). The six classes are identified by color: Red Ash above Sea. Cyan - Ash above meteorological clouds. Yellow - Meteorological clouds. Blue - Sea. White - Ice. Green Land. The coastline is plotted in black. Volcano's vents are marked by black stars.

\section{RESULTS}

Moreover, we have found that the inclusion of the MODIS L1 Land/Mask improves the generalization of the classifier. The final input space of the NN is therefore composed of 15 inputs, while the output space is composed of six classes provided by the standard procedure (see Fig. 2). By applying a simple trial and error procedure, the best topological performance proved to be the combination of two hidden layers with 15 and 5 neurons, respectively. In the NN learning phase, to avoid the oversampling of particular classes, the same number of pixels was randomly extracted for each class. As a result, for each one of the six classes 2660 pixels where extracted from the JD 126 image. The NN was trained using an error back-propagation algorithm on the training set (equal to $75 \%$ of the pixel considered from the JD 126 image). Using the cross validation technique the training was interrupted when the generalization performance evaluated on a different image subset (on the remaining $25 \%$ of pixels) degenerated.
The NN's performance has been evaluated by using the independent dataset of the MODIS images (JD 128, JD 132 and JD 133 from 2010 and JD 146 from 2011). The results are satisfactory with overall classification accuracy greater than $84 \%$ for the Eyjafjallajökull validation cases, and equal to $74 \%$ for the Grimsvötn image. Figure 2 shows the classified maps for all the MODIS acquisitions obtained by the benchmark "standard procedure" and the NN approach respectively. The figure shows how the standard products and the NN classification are qualitatively compatible. The quantitative analysis of the automatic algorithm's performance is given by the confusion matrixes for the validation dates presented in figure 3, where the Target Class refers to the "standard procedure" results and the Output Class to those of the NN's. The errors for each class are also given, as well as the commission and omission errors reported in the last grey column and row of each matrix [Richards and 
Jia, 1999]. In observing the main matrices diagonally it can be noted that the majority of the pixels belonging to every class have been correctly classified, even if a few classification errors are still present. These mainly occur between spatially or spectrally adjacent classes. Firstly, the "Ice" class is sometimes identified by the NN where the "standard procedure" indicates meteorological clouds. As described in Section II, in the "standard procedure" the meteorological clouds are classified by using the MODIS L2 Cloud product. In the event of semitransparent meteorological formation (thin clouds), the contribution of the underlying surface is not negligible and may strongly characterize the radiometric response. In these intermediate conditions pixel mis-classifications can occur. Secondly, some errors occur on ash over sea pixels that are classified as ash over clouds (i.e. in JD128 case). Again, this behavior seems to be due to the method used to define the classes in the "standard procedures". Indeed, to identify the ash over clouds and meteorological clouds, the MODIS L2 data are used to apply a cloud mask. This procedure does not ensure that one pixel identified as ash and not flagged as cloudy will not present mixed effects according to meteorological formations. On the contrary, it is reasonable that pixels identified as cloudy and free from ash, are partially contaminated by ash and thus classified as ash over cloud by the NN (i.e. in JD 132 case). Finally, considering the spatial distributions of the NN classified features (i.e. ash clouds, glaciers, meteorological clouds) it has to be underlined that the misclassification errors are mainly localized on the edges of correctly classified objects, and that the spatial features of the "standard procedure" and the NN classifier images are accordance with each other. This is also the reason why the Land class in JD 143 has very low clas- sification accuracy: it is represented by a very small number of pixels distributed on the borders of other classes.

\section{Conclusions}

This experiment demonstrates the NN's potentials in classifying eruptive event images. Despite some mis-classification errors, the proposed automatic classifier shows good overall accuracy, greater that $84 \%$ for the Eyjafjallajökull validation images. In the more challenging Grimsvötn case, data achieves an overall accuracy equal to $74 \%$, and a spatial distribution for the classified features consistent with the results obtained by the interactive and time consuming workflows. The trained NN's capability of immediately providing reliable classification maps of multispectral satellite images can be profitably used to improve ash retrieval parameters in an operational scenario and hereafter be applied to processing images of new eruptions, including those of other volcanoes in particular conditions (i.e. ash characteristics, latitude and season). Furthermore, to improve the NN's accuracy, additional data can be considered. However, this is not necessary if the training set is already statistically representative of the studied phenomena. Future developments could enhance the NN's potentials by improving its capability to detect other classes like "Ash on Land" and "Desert Dust" in different climate conditions.

\section{ACKNOWLEDGEMETS}

The work of S. Corradini was founded by the European Union's Seventh Framework Programme (FP7/2007-2013) through the project APhoRISM under grant agreement number 606738.

\section{REFERENCES}


[Bishop, 1995] Bishop C. M. (1995). Neural Networks for Pattern Recognition. Oxford Univ. Press.

[Chini et al., 2008] M. Chini, Pacifici, F., et al. (2008). Comparing Statistical and Neural Network Methods Applied to Very High Resolution Satellite Images Showing Changes in Man-Made Structures at Rocky Flats. IEEE Trans. Geosci. Remote Sens., 46(6), 1812- 1821.

[Corradini et al., 2008] Corradini, S., Spinetti, C. et al. (2008) Mt. Etna tropospheric ash retrieval and sensitivity analysis using Moderate Resolution Imaging Spectroradiometer measurements. J, Atmosph. Rem. Sens., 2, 023550.

[Corradini et al., 2010] Corradini, S., Merucci, L. et al. (2010). Volcanic ash and $\mathrm{SO}_{2}$ in the 2008 Kasatochi eruption: Retrievals comparison from different IR satellite sensors. J. Geophys. Res., 115(D2), D00L21.

[Picchiani et al., 2011] Picchiani, M., Chini, M., et al. (2011). Volcanic ash detection and retrievals from MODIS data by means of Neural Networks. Atmos. Meas. Tech., 4, 2619-2631.

[Piscini et al., 2014] Piscini, A., Picchiani, M., et al. (2014). A Neural Network approach for the simultaneous retrieval of volcanic ash parameters and $\mathrm{SO}_{2}$ using MODIS data. Atmos. Meas. Tech., 7, 4023-4047.

[Prata, 1989] Prata A. J. (1989). Infrared radiative transfer calculations for volcanic ash clouds. Geophys. Res. Lett., 16(11), 1293-1296. [Prata and Kerkmann, 2007] Prata, A. J., and Kerkmann, J. (2007). Simultaneous retrieval of volcanic ash and SO2 using MSG-SEVIRI measurements. Geophys. Res. Lett, 34(5), L05813.

[Richards and Jia, 1999] Richards, J.A., Jia, X. (1999). Remote Sensing Digital Image Analysis: An Introduction. New York: Springer-Verlag.
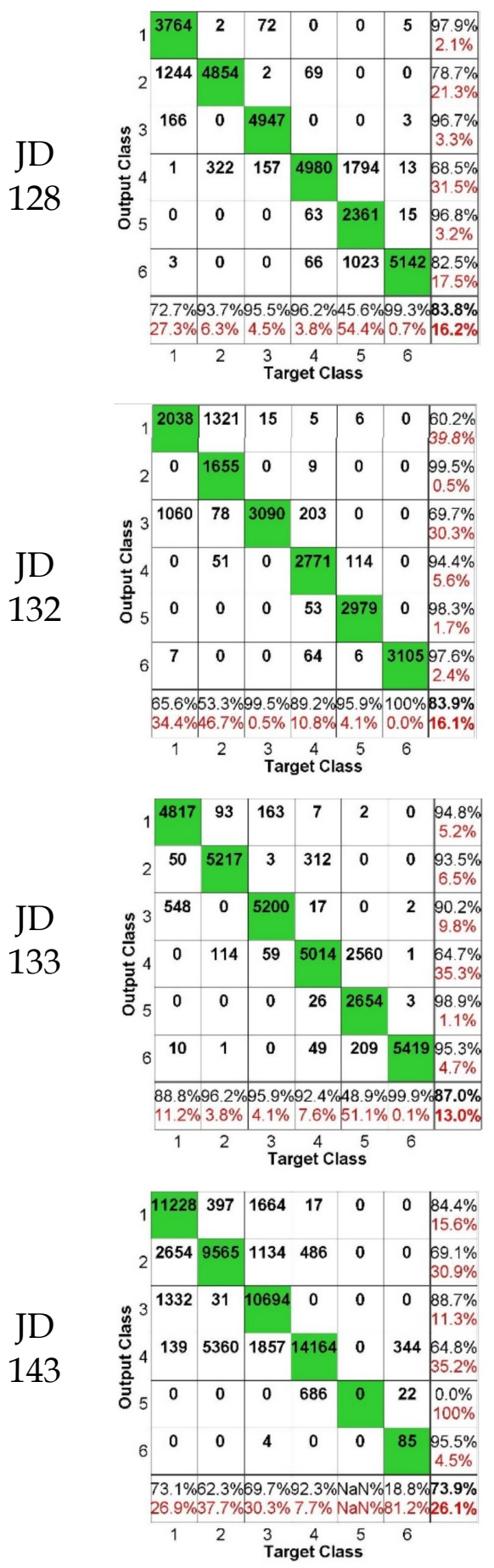

Figure 3: Confusion matrices. The six classes are: 1 Ash above Sea. 2 - Ash above meteorological clouds. 3 Meteorological clouds. 4 - Sea. 5 - Ice. 6 - Land. 